\title{
Análisis de las principales competencias necesarias para la implementación de la Industria 4.0 en el sector agroindustrial uruguayo
}

Analyzing the main competences required for the implementation of Industry 4.0 in the Uruguayan agro-industry sector

Resumen. -En paralelo con los desafíos técnicos relacionados a la Industria 4.0, un nuevo reto surge: ¿cómo preparar a los actuales y futuros trabajadores, de manera de que puedan adaptarse a los empleos que surgirán y que demandarán nuevos requisitos en términos de competencias? El objetivo principal de este proyecto es investigar qué competencias serán esenciales para el futuro del sector agroindustrial. Se enfoca el estudio en dicho sector dado que este es uno de los sectores económicos con mayor riesgo de automatización de los puestos de trabajo según la literatura. Para determinar las competencias necesarias, se realizó una extensa revisión de la literatura, de la que surgieron 31 competencias consideradas fundamentales para el futuro. Dicha matriz de competencias fue luego validada a través de la realización de una serie de entrevistas semi-estructuradas a stakeholders del sector, para determinar los niveles actuales en materia de esas 31 competencias, y su ponderación para el futuro. En particular, se generaron gráficos de radar mostrando los valores actuales y futuros, identificando el gap de competencias necesario para avanzar en materia de transformación digital y tecnológica del sector. Las competencias determinadas como las más relevantes para el futuro son: Conocimientos de Informática, Capacidad de operar con nuevas interfaces, Aprendizaje Continuo, Pensamiento Analítico y Lógico, Expresión Oral, Trabajo en equipo, Confianza en la tecnología y Flexibilidad

Palabras Clave: Industria 4.0, agroindustria, competencias profesionales, factor humano

Abstract. -In parallel with the technical challenges related to Industry 4.0, a challenge arises about how to prepare current and future workers so that they can adapt to future jobs, which will demand new requirements in terms of competences and skills. The main objective of this paper is to investigate which competences will be essential towards the future jobs in the agro-industry sector. The investigation was focused in this sector, since is one of the economic sectors with the greatest risk of job automation. An extensive literature review, followed by in depth interviews to relevant stakeholders in the agricultural sector in Uruguay were conducted in order to identify the main competences considered critical towards the future. In particular, radar graphs were created, showing the actual and expected level of development of 31 professional competences, divided into four main categories. The competences acknowledged by the stakeholders as most relevant for the future are: IT knowledge, Ability to interact with modern interfaces, Active Learning, Analytical and Logical Thinking, Oral expression, Teamwork, Trust in technology and Flexibility.

Keywords: Industry 4.0, agribusiness, professional competences, human factor

\footnotetext{
${ }^{1}$ CINOI - Universidad de Montevideo; djurburg1@um.edu.uy ORCID 0000-0001-9226-1520

${ }^{2}$ CINOI - Universidad de Montevideo. ORCID 0000-0001-8646-7709
} 
1. Introducción. -El mundo se ha enfrentado a momentos en los que la tecnología disruptiva modifica la forma en que operan las fábricas y las empresas, y, por lo tanto, también lo hicieron las competencias que los trabajadores necesitaban para tener éxito. Estos cambios repentinos suelen denominarse revolución industrial. Tres de ellas ya transcurrieron, y una Cuarta Revolución, también conocida como Industria 4.0, está ocurriendo en estos momentos en que la tecnología de la digitalización y la robótica están aumentando [1]. Esta nueva revolución, como indican muchos estudios internacionales, generará en un futuro cercano una gran disrupción de las competencias laborales necesarias, y ese será uno de los principales desafíos a afrontar [2-7].

Además, esta revolución está conduciendo a automatizar gran parte de la producción, con una consecuencia evidente: una gran cantidad de puestos de trabajo pueden desaparecer. Por ejemplo, un estudio realizado por Frey y Osborne [5] concluyó que el $47 \%$ del empleo total en Estados Unidos tiene un alto riesgo de automatización en un lapso de 20 años. Este estudio indica, a su vez, que una de las áreas de producción que tiene el mayor riesgo de automatizarse es la agrícola. Este sector presenta una fuerza de trabajo con un nivel educativo bajo -en rasgos generales-, que mayormente realiza tareas manuales y repetitivas. Esto hace que sea un sector con alto riesgo de automatización y de difícil reconversión laboral.

Por dicho motivo, este proyecto tiene como objetivo principal identificar las principales competencias necesarias para el sector agroindustrial en el futuro de la Industria 4.0. Este tipo de investigaciones les permitirá a las empresas obtener información científica que las habilite a comenzar a diseñar e implementar programas de capacitación para sus empleados, y mejorar así su idoneidad frente a esta revolución.

2. Industria 4.0. -Este término fue presentado en la Feria de Hannover en el año 2011. Describía la iniciativa común entre empresas, educadores y gobierno para fortalecer la competitividad de la industria manufacturera alemana. Más recientemente, varios países han adoptado también un abordaje similar para el futuro [4, 8]. Dado que este concepto es muy reciente, aún no se ha propuesto una definición única [9]. Igualmente, la mayor parte de las definiciones coinciden en algunos aspectos en común. Una característica principal es la integración del mundo físico y digital, permitiendo el intercambio de datos en tiempo real entre personas, máquinas y productos, a lo largo de toda la cadena de valor. En este sentido, aparecen tres componentes que se consideran críticos: el Internet de las Cosas, los Sistemas Ciberfísicos, y las Fábricas Inteligentes [9]. El primero de ellos permite que las máquinas, productos y objetos interactúen entre sí y cooperen para lograr sus objetivos. El segundo permite la fusión entre el mundo real y el virtual, mediante sistemas de computación embebidos en los elementos de producción, que monitorean y controlan los procesos. Y el tercer componente es la combinación de las dos tecnologías anteriores para lograr un sistema de producción más descentralizado y autónomo.

Uno de los principales desafíos que se derivan de esta nueva revolución industrial es lo referente a los puestos de trabajo. Algunos reportes sugieren que van a disminuir [10], mientras que otros afirman que es más probable que la creación de trabajos exceda a aquellos destruidos [3]. Varios estudios refieren a esta dicotomía y concluyen que ambos futuros son posibles, y solo el tiempo dirá cuál va a ocurrir [11]. En lo que la mayoría de las investigaciones están de acuerdo es que los trabajadores van a tener que desarrollar nuevas competencias para poder adaptarse correctamente a los cambios que propondrá la Industria 4.0.

Memoria Investigaciones en Ingeniería, 2019. $\mathrm{N}^{\circ} 17(1) . p p$ 151-171

https://doi.org/10.36561/ING.17.7

ISSN 2301-1092・ISSN (en línea) 2301-1106 
3. Importancia del sector Agroindustrial. -De acuerdo al Banco Mundial [12], el sector agroindustrial es crucial para el crecimiento económico de las naciones, ya que representó un tercio del producto bruto interno mundial en el año 2014. A su vez, se refieren a este sector como una de las mejores herramientas para terminar la pobreza extrema y lograr alimentar a las 9.7 billones de personas esperadas para el 2050. También indican que actualmente un $25 \%$ de la población mundial trabaja en este sector. Estos números muestran el panorama de crucial importancia que reviste este sector para el mundo entero; por un lado, por la necesidad de un crecimiento en la producción de alimentos, y, por otro lado, debido a la cantidad de gente que emplea.

Particularmente en Uruguay, la agroindustria es un motor central de la economía, representando el 79\% del valor de las exportaciones en el 2017 [13]. Cabe destacar a su vez, que de las 20 empresas que más exportaron en el 2017, 17 fueron empresas agroindustriales [14]. Aproximadamente el 15\% de la mano de obra del país se encuentra en esta industria. Pero esta mano de obra tiene un bajo nivel de instrucción, en términos generales. Solamente el 4\% tiene un nivel terciario o mayor [15]. Esto es una limitación para el sector, y una dificultad que deberá ser enfrentada por cualquiera que esté dispuesto a entrenar trabajadores en esta área.

Habiendo apreciado la importancia de este sector y la cantidad de empleos que representa a nivel mundial, su futuro en la Industria 4.0 debe ser analizado cuidadosamente, en especial considerando que los estudios existentes en la literatura proponen al sector como uno de los que tienen los mayores riesgos de automatización. Por ejemplo, Manyika et al. [2] señala que es el tercer sector económico con riesgo más alto de automatización, con un 58\%, mientras que un estudio en Uruguay le da a este sector el mayor riesgo, con 78\% [16]. Estos resultados soportan la necesidad de seguir investigando en este sector, en particular trabajando para identificar cuáles son las competencias que serán necesarias en este sector en el futuro, de manera de minimizar el impacto negativo que la transformación 4.0 pueda generar en las personas empleadas en él.

4. Metodología. -Para poder identificar las competencias que serán cruciales en el sector agroindustrial para la aplicación de la Industria 4.0, se realizó una extensa revisión bibliográfica, seguida de una serie de entrevistas semi-estructuradas a una muestra seleccionada dentro de un grupo de stakeholders del sector agropecuario uruguayo. La metodología se desarrolló como se indica a continuación.

4.1 Revisión literaria. -En primer lugar, se realizó una detallada revisión de la literatura para definir las principales competencias consideradas esenciales para el futuro de la Industria 4.0. La revisión se realizó inicialmente en Scopus, al ser una de las mayores y más prestigiosas bases de datos de revisión por pares, y luego se amplió con otras bases de datos.

La primera búsqueda efectuada fueron las palabras "Industry 4.0" y "Fourth Industrial Revolution". De esta búsqueda se obtuvieron 6.083 resultados, lo que muestra la cantidad de trabajos vinculados a esta temática. De todos estos resultados, más del $85 \%$ son posteriores al año 2010, lo que refleja la actualidad de la misma. Posteriormente, dada la gran cantidad de resultados obtenidos, se realizó un refinamiento de la búsqueda agregando las palabras "skills", "qualification", "employment", "human resources" y "abilities".

La búsqueda presentada en la Tabla I, resulta:

Memoria Investigaciones en Ingeniería, 2019. $\mathrm{N}^{\circ} 17(1) . p p$ 151-171

https://doi.org/10.36561/ING.17.7

ISSN 2301-1092 • ISSN (en línea) 2301-1106 


\begin{tabular}{|l|l|l|}
\hline Industry 4.0 & & Skills \\
& & OR \\
& & Abilities \\
& ORD & OR \\
& & Human Resources \\
& & OR \\
Fourth Industrial Revolution & & Qualification \\
& & OR \\
& & Employment \\
\hline
\end{tabular}

Tabla I: Esquema de la revisión bibliográfica realizada en Scopus

Este refinamiento brindó 639 documentos relevantes, de los cuáles 38 eran en idiomas diferentes al inglés o español, por lo que fueron inmediatamente excluidos. Se procedió posteriormente con una lectura de todos los títulos, y en aquellos que resultaban acordes a la temática se procedía a una lectura completa del resumen. De este análisis se eligieron 39 artículos que revestían una vinculación estrecha con el concepto de Industria 4.0, si bien no necesariamente hacían referencia explícita al tema de competencias necesarias.

A continuación, se decidió ampliar el espectro de búsqueda para encontrar otros trabajos de relevancia fuera de Scopus. La herramienta principal fue Google, y las palabras claves fueron las mismas que en la búsqueda previa. De esto surgieron trabajos muy interesantes, incluyendo artículos y reportes de las siguientes fuentes:

- UK Commission for Employment and Skills

- United Nations Educational, Scientific and Cultural Organization

- American Society of Mechanical Engineers

- BRICS Skill Development Working Group

- European Parliament's Committee on Industry, Research and Energy (ITRE)

- Deloitte

- Word Economic Forum

- McKinsey

- Boston Consulting Group

En total, 69 trabajos fueron seleccionados y analizados. De estos, solo 20 trabajos abordaban de forma más detallada y explícita aspectos referentes a las competencias necesarias para la Industria 4.0.

Luego de una caracterización general de los 20 trabajos seleccionados para el análisis, se procedió a realizar una lectura profunda de cada uno de ellos, extrayendo de los mismos las principales competencias mencionadas en cada artículo como fundamentales para el futuro y se las categorizó de acuerdo al marco conceptual propuesto por Hecklau et al. [17], que propone cuatro grandes categorías que son: Competencias Técnicas, Competencias Metodológicas, Competencias Sociales y Competencias Personales. Para ello, se generaron tablas con todas las competencias mencionadas por cada una de las cuatro categorías ya indicadas, y luego se tabuló la frecuencia de repetición de las mismas en la literatura analizada.

4.2 Entrevistas. -Para lograr contextualizar la realidad de la Industria 4.0 y las competencias necesarias para ella dentro del sector agroindustrial uruguayo, se decidió validar la matriz de competencias generada a través de la realización de una serie de entrevistas semi-estructuradas a empresas y organismos referentes del sector ${ }^{3}$. Se les

\footnotetext{
${ }^{3}$ El cuestionario completo utilizado se puede solicitar a los autores

Memoria Investigaciones en Ingeniería, 2019. $\mathrm{N}^{\circ} 17(1) . p p$ 151-171

https://doi.org/10.36561/ING.17.7

ISSN 2301-1092・ISSN (en línea) 2301-1106
} 
solicitaba a los entrevistados, entre otras cosas, que evaluaran, para cada una de las competencias anteriores, de 1 a 5 (siendo 1 muy poco capacitado, y 5 muy capacitado) el nivel actual que identificaban en los operarios del sector, y en nivel que consideraban que iba a ser necesario que tuvieran en 10 años.

El formato utilizado para las entrevistas se basó en los trabajos previos de Lleó et al. [18] y Hamzeh et al. [6], teniendo como principal objetivo identificar el gap de conocimiento al que los trabajadores de la agroindustria deberán enfrentarse ante el arribo de las nuevas tecnologías y modelos de negocio. Los resultados de dichas entrevistas se plasmaron en gráficos de radar, los cuales indican el nivel de competencias que los empleadores perciben en los trabajadores de la agroindustria hoy en día, y el nivel que consideran será necesario que tengan dentro de 10 años. A su vez, de las entrevistas se pretendió determinar, según la visión de los entrevistados, cómo la universidad puede colaborar con las empresas en los cambios tecnológicos que se están llevando a cabo. Y también se pretendió evaluar según la visión de las empresas del sector, la disrupción de los puestos de trabajo que esta tecnología traerá aparejada.

Previo a la realización de las entrevistas, se planteó un análisis de los stakeholders del sector agropecuario del país, de forma de identificar cuáles de ellos iban a ser más relevantes en las entrevistas. A su vez, se pretendió que todos los agentes interesados dentro del sector formaran parte de los entrevistados. Las categorías que se identificaron dentro de los stakeholders fueron: Productores (en donde están incluidas empresas de los rubros Forestal, Ganadería y Agricultura), Tecnología (implicando las empresas desarrolladoras de software para el agro, y las empresas proveedoras de tecnología para el mismo sector), Institutos Educativos y Gobierno (instituciones de gobierno enfocadas en el sector agropecuario). Esta división se aprecia en la Figura I.

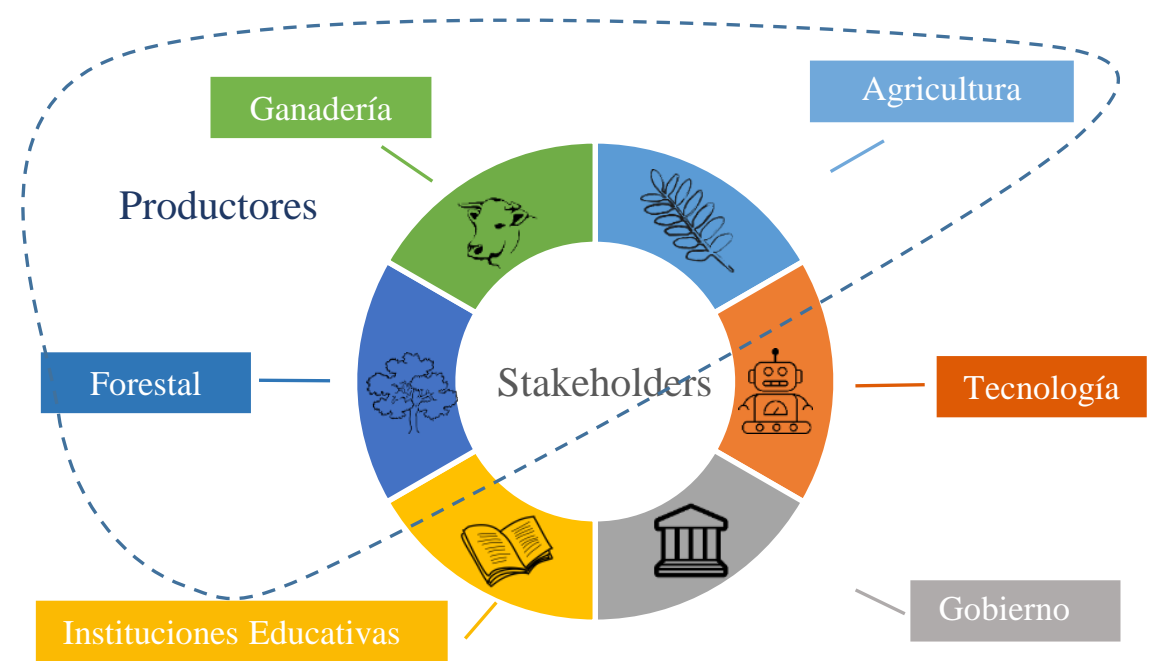

Figura I: Stakeholders del sector agropecuario uruguayo

En total se realizaron 15 entrevistas que tuvieron una duración media de 45 minutos cada una. Dentro de la categoría denominada "Productores", se realizaron 7 entrevistas. De ellas, 5 se realizaron a empresas que se encuentran dentro de las 8 mayores empresas exportadoras del país en el año 2017 (pertenecientes todas al sector agropecuario). Esas 5 empresas representaron aproximadamente un quinto del total de exportaciones del país en dicho año. Tanto dentro de la categoría "Tecnología" como "Instituciones Educativas" se realizaron 3 entrevistas en cada una, y dentro de "Gobierno" fueron realizadas otras 2 entrevistas. Las personas entrevistadas fueron en su gran mayoría gerentes generales, gerentes de producción, gerentes de recursos humanos y gerentes de mejora continua. De

Memoria Investigaciones en Ingeniería, 2019. $\mathrm{N}^{\circ} 17(1)$. $p p$ 151-171

https://doi.org/10.36561/ING.17.7

ISSN 2301-1092 • ISSN (en línea) 2301-1106 
esta forma se procuró entrevistar a las personas con más conocimiento y experiencia en la empresa acerca de las nuevas tecnologías y los consecuentes requerimientos de competencias en sus trabajadores.

\section{Resultados}

5.1 Generación de la tabla de competencias. -Para determinar las competencias necesarias para el futuro, se trabajó en base a la revisión bibliográfica explicada anteriormente. Los artículos analizados se presentan en la Tabla II, estando numerados del mismo modo en que aparecerán luego en las tablas de competencias.

\begin{tabular}{|l|l|l|l|}
\hline $\begin{array}{l}\text { 1.World Economic } \\
\text { Forum [19] }\end{array}$ & 6. Smit et al. [21] & 11. Moldovan [25] & 16. Scott [27] \\
$\begin{array}{l}\text { 2. World Economic } \\
\text { Forum [10] }\end{array}$ & 7. Erol et al. [22] & 12. Störmer et al. (26) & 17. Kergroach [28] \\
\hline $\begin{array}{l}\text { 3. Benešová \& Tupa } \\
\text { [1] }\end{array}$ & 8. Breuer [23] & $\begin{array}{l}\text { 13. Hecklau, et al. } \\
\text { [17] }\end{array}$ & 18. Lorenz et al. [29] \\
\hline $\begin{array}{l}\text { 4. Gehrke et al. [20] } \\
\text { 9. European Centre for } \\
\text { the Development of } \\
\text { Vocational Training [24] }\end{array}$ & $\begin{array}{l}\text { 14. Lleó et al. [18] } \\
\text { 10. Aulbur et al. [11] }\end{array}$ & $\begin{array}{l}\text { 19. Cotet et al [30] } \\
\text { Cortelezzi [15] }\end{array}$ & 20. Davies et al. [31] \\
\hline 5. Lorenz et al. [7] & 19. &
\end{tabular}

Tabla II: Artículos analizados para determinar las competencias frente a la Industria 4.0.

A los efectos de realizar una breve caracterización de los trabajos analizados, al agrupar estos 20 estudios, se advierten las siguientes características:

- El 85\% de ellos tienen menos de 4 años de publicación, lo que muestra que el interés sobre esta temática es muy reciente. Esto es un signo que el interés está creciendo gradualmente, y en los siguientes años la literatura sobre esta temática posiblemente seguirá con un ritmo de crecimiento aún mayor.

- Acerca del origen de los trabajos, la gran mayoría son de Europa, destacándose la participación de Alemania en estas investigaciones, motivo que no es casual dado que el origen de este concepto pertenece al gobierno de dicho país.

- Sobre la metodología, cabe destacar que 8 trabajos se basaron en la realización de encuestas. Los países en los que se realizaron dichas encuestas fueron Australia, Brasil, China, Francia, Alemania, Estados Unidos, India, Italia, Japón, México, por destacar algunos. En conjunto, estas entrevistas abarcan más de 1.300 empresas, que emplean alrededor de 30 millones de personas. Las mayores encuestas se dieron en años recientes y buscan recabar datos para tratar de comprender en mayor profundidad qué efectos tendrá la aplicación de estas tecnologías en las empresas [7, 10, 19]. Por lo tanto, se tiene información abundante sobre esta temática, mostrando las experiencias y estimaciones de las principales empresas del mundo. Otra metodología aplicada en estos trabajos fue la realización de un estudio teórico, analizando datos recabados de distintas fuentes para estimar principalmente qué capacidades van a ser fundamentales en un futuro, y como se verán afectados los trabajadores. Por último, 3 trabajos se basaron esencialmente en la realización de una revisión literaria para aunar los datos de diversas fuentes.

Como fuera explicado anteriormente, para determinar las competencias necesarias para el futuro, se trabajó en base a estos 20 trabajos. Para ello, se extrajeron las principales

Memoria Investigaciones en Ingeniería, 2019. $\mathrm{N}^{\circ} 17(1)$. pp 151-171

https://doi.org/10.36561/ING.17.7

ISSN 2301-1092・ISSN (en línea) 2301-1106 
competencias que menciona cada artículo como fundamental para el futuro y se las agrupó en las 4 categorías mencionadas anteriormente.

Las tablas resultantes (III, IV, V y VI) y las competencias más destacadas se presentan a continuación.

Competencias técnicas: en primer lugar, se encuentra conocimientos de informática. Luego se encuentra la programación, y en tercer lugar hay varias competencias con una similar cantidad de menciones. Ellas son media- skills, procesamiento y análisis de datos, comprensión de los procesos y la organización y concientización sobre seguridad informática. A su vez, es interesante destacar que la gran mayoría de las competencias técnicas referidas internacionalmente están estrechamente vinculadas al uso de herramientas de informática y a las nuevas tecnologías que destacarán en el futuro.

\begin{tabular}{|c|c|c|c|c|c|c|c|c|c|c|c|c|c|c|c|c|c|c|c|c|c|}
\hline $\begin{array}{l}\text { Competencias } \\
\text { técnicas }\end{array}$ & 1 & 2 & 3 & 4 & 5 & 6 & 7 & 8 & 9 & 10 & 11 & 12 & 13 & 14 & 15 & 16 & 17 & 18 & 19 & 20 & Tot \\
\hline Programación & $\checkmark$ & $\checkmark$ & $\checkmark$ & $\checkmark$ & $\checkmark$ & & & & $\checkmark$ & & & & $\checkmark$ & $\checkmark$ & & & $\checkmark$ & $\checkmark$ & & & 10 \\
\hline $\begin{array}{l}\text { Control y operación de } \\
\text { equipos }\end{array}$ & & $\checkmark$ & & & $\checkmark$ & & $\checkmark$ & & & $\checkmark$ & & & & $\checkmark$ & & & & & & & 5 \\
\hline Media Skills & & $\checkmark$ & $\checkmark$ & & & & $\checkmark$ & $\checkmark$ & & & & $\checkmark$ & $\sqrt{ }$ & & & & & & & $\sqrt{ }$ & 7 \\
\hline $\begin{array}{l}\text { Conocimientos de } \\
\text { informática }\end{array}$ & & $\checkmark$ & $\checkmark$ & $\checkmark$ & & & $\checkmark$ & & $\checkmark$ & $\checkmark$ & & $\checkmark$ & & $\checkmark$ & $\checkmark$ & $\checkmark$ & $\checkmark$ & $\checkmark$ & & & 12 \\
\hline $\begin{array}{l}\text { Procesamiento y análisis } \\
\text { de datos }\end{array}$ & & $\checkmark$ & $\checkmark$ & $\checkmark$ & $\checkmark$ & & $\checkmark$ & & & & & & & $\checkmark$ & & $\checkmark$ & & & & $\checkmark$ & 8 \\
\hline $\begin{array}{l}\text { Comprensión de los } \\
\text { procesos y la } \\
\text { organización }\end{array}$ & & & & $\checkmark$ & & & $\checkmark$ & & & & & $\checkmark$ & $\checkmark$ & $\checkmark$ & & & & & & & 5 \\
\hline $\begin{array}{l}\text { Capacidad de operar con } \\
\text { nuevas interfaces }\end{array}$ & & & $\checkmark$ & $\checkmark$ & $\checkmark$ & & & & & $\checkmark$ & & & & $\checkmark$ & & & & & & & 5 \\
\hline $\begin{array}{l}\text { Concientización sobre } \\
\text { seguridad informática }\end{array}$ & & & $\checkmark$ & $\checkmark$ & $\checkmark$ & & $\checkmark$ & & & $\checkmark$ & & & $\checkmark$ & $\checkmark$ & & & & & & & 7 \\
\hline
\end{tabular}

Tabla III: Tabla de competencias técnicas

Competencias metodológicas: la más mencionada es la necesidad de un aprendizaje continuo. Le siguen el pensamiento analítico y lógico y resolución de problemas. Y también se destacan repetidas veces la innovación y la creatividad. El foco en la necesidad de un aprendizaje continuo muestra que en el futuro los trabajadores deberán estar formándose continuamente para seguir dentro del mercado laboral. La tecnología hará que los cambios se den cada vez con más rapidez y será necesario estar constantemente actualizándose acerca de los mismos para poder aportar en el empleo. 


\begin{tabular}{|c|c|c|c|c|c|c|c|c|c|c|c|c|c|c|c|c|c|c|c|c|c|}
\hline $\begin{array}{l}\text { Competencias } \\
\text { metodológicas }\end{array}$ & 1 & 2 & 3 & 4 & 5 & 6 & 7 & 8 & 9 & 10 & 11 & 12 & 13 & 14 & 15 & 16 & 17 & 18 & 19 & 20 & Tot \\
\hline $\begin{array}{l}\text { Pensamiento analítico y } \\
\text { lógico }\end{array}$ & $\sqrt{ }$ & $\checkmark$ & $\sqrt{ }$ & & $\checkmark$ & $\sqrt{ }$ & $\checkmark$ & & & & $\checkmark$ & & $\checkmark$ & & $\sqrt{ }$ & $\sqrt{ }$ & & & & $\sqrt{ }$ & 11 \\
\hline Innovación & $\checkmark$ & $\checkmark$ & $\sqrt{ }$ & & & & $\checkmark$ & $\checkmark$ & $\checkmark$ & $\checkmark$ & & & $\checkmark$ & & & $\checkmark$ & & $\sqrt{ }$ & & & 10 \\
\hline Aprendizaje Continuo & $\checkmark$ & $\checkmark$ & $\checkmark$ & $\checkmark$ & & & $\checkmark$ & $\checkmark$ & $\checkmark$ & $\checkmark$ & & $\checkmark$ & $\checkmark$ & & & $\checkmark$ & $\sqrt{ }$ & $\checkmark$ & & $\sqrt{ }$ & 14 \\
\hline Creatividad & $\sqrt{ }$ & $\checkmark$ & $\sqrt{ }$ & & & & $\checkmark$ & & & & $\checkmark$ & & $\checkmark$ & & & $\checkmark$ & $\sqrt{ }$ & & $\checkmark$ & $\sqrt{ }$ & 10 \\
\hline Resolución de problemas & $\sqrt{ }$ & $\checkmark$ & $\checkmark$ & & & $\checkmark$ & $\checkmark$ & $\checkmark$ & $\checkmark$ & & $\checkmark$ & & $\checkmark$ & & & $\checkmark$ & $\checkmark$ & & & & 11 \\
\hline Emprendedurismo & & & & & & & $\checkmark$ & & & & & & & & & & & & & & 1 \\
\hline $\begin{array}{l}\text { Habilidades de } \\
\text { investigación }\end{array}$ & & & & & & & & & & & & & $\checkmark$ & & & & & & & & $\mathbf{1}$ \\
\hline Transdisciplinariedad & & & & & & & $\checkmark$ & & & & & & & & & & & & & $\checkmark$ & 2 \\
\hline
\end{tabular}

Tabla IV: Tabla de competencias metodológicas

Competencias sociales: hay dos que sobresalen por encima del resto. Estas son trabajo en equipo y capacidad de buena comunicación. En el futuro los empleos metódicos estarán realizados casi exclusivamente por tecnología, por lo que el aporte de las personas será en actividades menos automatizables, que dependan mucho de la relación entre pares. Por dicho motivo es que el trabajo en equipo se destaca, y para que esta relación sea buena y brinde beneficios debe estar cimentada por unas buenas habilidades de comunicación.

\begin{tabular}{|c|c|c|c|c|c|c|c|c|c|c|c|c|c|c|c|c|c|c|c|c|c|}
\hline $\begin{array}{l}\text { Competencias } \\
\text { sociales }\end{array}$ & 1 & 2 & 3 & 4 & 5 & 6 & 7 & 8 & 9 & 10 & 11 & 12 & 13 & 14 & 15 & 16 & 17 & 18 & 19 & 20 & Tot \\
\hline Inteligencia emocional & $\checkmark$ & $\checkmark$ & & & & & & & & & $\checkmark$ & $\checkmark$ & & & & & & & & $\checkmark$ & 5 \\
\hline $\begin{array}{l}\text { Capacidad de buena } \\
\text { comunicación }\end{array}$ & & $\checkmark$ & $\checkmark$ & $\checkmark$ & & $\sqrt{ }$ & $\sqrt{ }$ & & $\checkmark$ & $\checkmark$ & $\checkmark$ & $\sqrt{ }$ & $\sqrt{ }$ & $\sqrt{ }$ & & $\checkmark$ & $\checkmark$ & & & & 13 \\
\hline $\begin{array}{l}\text { Entrenar y enseñar a } \\
\text { otros }\end{array}$ & & $\sqrt{ }$ & & & & & & & & & & $\sqrt{ }$ & $\checkmark$ & & & & & & & & 3 \\
\hline Trabajo en equipo & & $\checkmark$ & $\checkmark$ & $\checkmark$ & & & $\sqrt{ }$ & $\checkmark$ & $\checkmark$ & $\checkmark$ & & $\sqrt{ }$ & $\sqrt{ }$ & $\checkmark$ & $\checkmark$ & $\checkmark$ & $\checkmark$ & & & $\sqrt{ }$ & 14 \\
\hline $\begin{array}{l}\text { Habilidades } \\
\text { Interculturales }\end{array}$ & & & & & & & $\checkmark$ & & & & & & $\checkmark$ & & & $\checkmark$ & & & & $\checkmark$ & 4 \\
\hline
\end{tabular}

Tabla V: Tabla de competencias sociales

Competencias personales: en primer lugar, nos encontramos con la habilidad de adaptarse a cambios. Luego aparecen destacadas también la responsabilidad y el liderazgo. Estas competencias se aúnan con las anteriores. Por un lado, la capacidad de adaptarse a cambios está íntimamente relacionada con el aprendizaje continuo. Para lograr adaptarse y seguir dentro del mercado laboral, lo fundamental es estar continuamente estudiando y manteniéndose al tanto de las nuevas innovaciones. A su vez, los desafíos por la incorporación de tecnología harán que los trabajos cambien y que se creen empleos que actualmente no existen. En ese momento el dilema será principalmente si quedar fuera del mercado laboral o reconvertirse para acceder a posiciones que aún no se hayan automatizado. Y, por otro lado, el liderazgo es una competencia estrictamente de las personas, que no es automatizable. Por dicho motivo es que se considera tan importante. 


\begin{tabular}{|c|c|c|c|c|c|c|c|c|c|c|c|c|c|c|c|c|c|c|c|c|c|}
\hline $\begin{array}{l}\text { Competencias } \\
\text { personales }\end{array}$ & 1 & 2 & 3 & 4 & 5 & 6 & 7 & 8 & 9 & 10 & 11 & 12 & 13 & 14 & 15 & 16 & 17 & 18 & 19 & 20 & Tot \\
\hline Iniciativa & $\checkmark$ & & $\checkmark$ & & & $\checkmark$ & & & & & & $\checkmark$ & & & & & & & & & 4 \\
\hline Liderazgo & $\checkmark$ & & & & & & & $\checkmark$ & & & & $\checkmark$ & $\checkmark$ & $\checkmark$ & & $\checkmark$ & & & & & 6 \\
\hline Fuerza física & & & & & & & & & & & & & & & & & & & & & 0 \\
\hline Habilidad manual & & & $\checkmark$ & & & & & & & & & & & & & & & & & & 1 \\
\hline $\begin{array}{l}\text { Buena administración } \\
\text { del tiempo }\end{array}$ & & $\checkmark$ & & $\checkmark$ & & $\checkmark$ & & & & & $\checkmark$ & $\checkmark$ & & & & & & & & & 5 \\
\hline Responsabilidad & & & $\checkmark$ & & & & & & & & & $\checkmark$ & & $\checkmark$ & $\checkmark$ & $\checkmark$ & $\checkmark$ & & $\checkmark$ & & 7 \\
\hline $\begin{array}{l}\text { Habilidad de } \\
\text { adaptarse a cambios }\end{array}$ & & & $\checkmark$ & $\checkmark$ & & & $\checkmark$ & & & $\checkmark$ & $\checkmark$ & $\checkmark$ & $\checkmark$ & $\checkmark$ & & $\checkmark$ & & $\checkmark$ & $\checkmark$ & & 11 \\
\hline $\begin{array}{l}\text { Confianza en la } \\
\text { tecnología }\end{array}$ & & & & $\checkmark$ & & & $\checkmark$ & & & & & $\checkmark$ & & $\checkmark$ & $\checkmark$ & & & & & & 5 \\
\hline $\begin{array}{l}\text { Interés en el } \\
\text { desarrollo personal }\end{array}$ & & & & & & & $\checkmark$ & & & & & $\checkmark$ & & & & & & & & & 2 \\
\hline Toma de decisiones & & & & & & & & & & & & & $\checkmark$ & $\checkmark$ & & & & & & & 2 \\
\hline
\end{tabular}

\subsection{Principales resultados de las entrevistas realizadas}

5.2.1 Matriz de competencias. -A partir de las entrevistas, se recopilaron todas las respuestas y se generaron distintos gráficos de radar con el fin de lograr identificar el gap de conocimiento que, en opinión de los entrevistados, presentan los trabajadores frente a la llegada de las nuevas tecnologías. Es claro que, si bien la metodología está fundamentada en la opinión de los entrevistados, y, por tanto, puede presentar ciertos sesgos naturales, de todas maneras, resulta útil para obtener las tendencias y comparar resultados acerca de qué competencias podrían ser más importantes frente al futuro, y dónde estarían presentes los principales gaps de conocimiento.

A su vez, en la entrevista se les solicitaba que, dentro de cada una de las cuatro categorías de competencias definidas previamente, identificaran en qué competencias centrarían los esfuerzos de capacitación dentro de la empresa en primer, segundo y tercer lugar si debieran de capacitar a sus trabajadores para el futuro. De esta forma se procuró detectar las competencias que los encuestados consideran, a priori, más relevantes para comenzar a capacitar a sus trabajadores de cara al futuro.

De forma de poder ponderar las competencias que los encuestados consideran más relevantes, se le planteó darle los valores que se indican en la Tabla VII, basados en trabajos anteriores

\begin{tabular}{|l|l|}
\hline Prioridad de capacitación & Valor de ponderación \\
\hline Capacitar en primer lugar & 5 \\
\hline Capacitar en segundo lugar & 3 \\
\hline Capacitar en tercer lugar & 1 \\
\hline
\end{tabular}

Tabla VII: Ponderación realizada para analizar las respuestas de las entrevistas

A continuación, se presentan los resultados obtenidos en base a las entrevistas. 
Competencias Técnicas. La Figura II presenta los resultados de las competencias técnicas actuales del operario en el sector agroindustrial, y el nivel que deberían tener dentro de 10 años, de acuerdo a la visión de los encuestados.

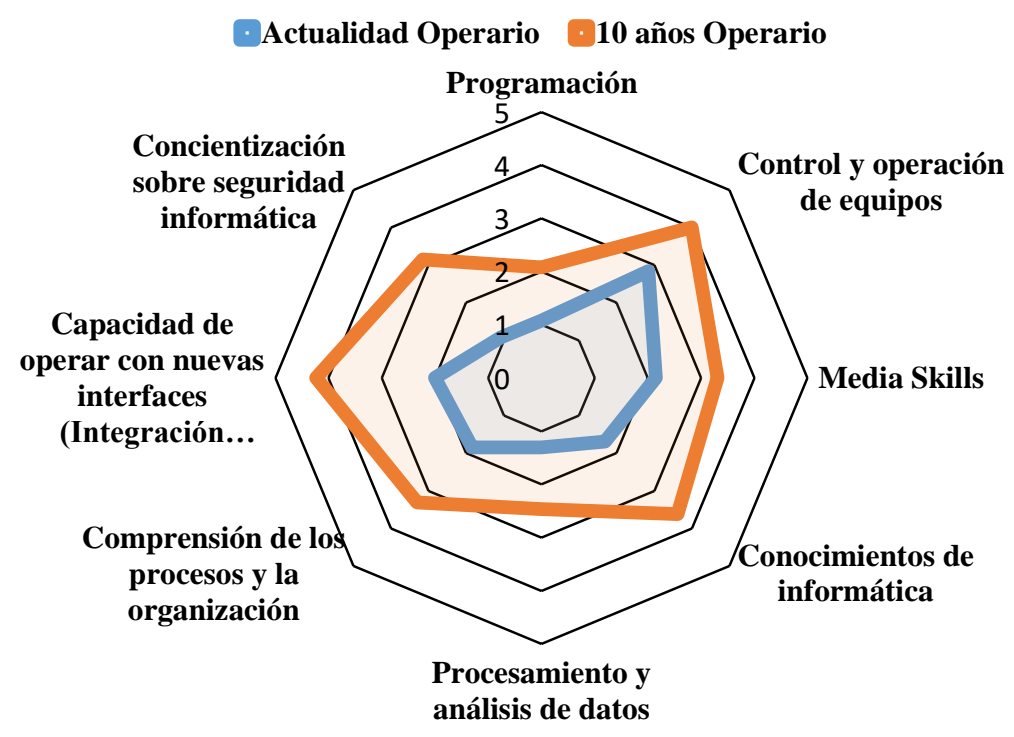

Figura II: Matriz de las competencias técnicas de los operarios

Para poder visualizar mejor la diferencia entre la actualidad y dentro de 10 años, se presenta en la Tabla VIII el valor de dicha diferencia. Las 3 mayores diferencias se destacan con color verde. Y a su vez, se presenta también el puntaje de ponderación obtenido por cada competencia para determinar aquellas que los empresarios consideran deberían ser las primeras en enfocarse. Se destacan con azul las 3 priorizadas.

\begin{tabular}{|c|c|c|c|}
\hline & & $\begin{array}{c}\text { Diferencia } \\
\text { Actualidad y } \\
10 \text { años } \\
\end{array}$ & Ponderación \\
\hline \multirow{8}{*}{ 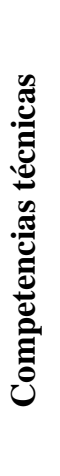 } & Programación & 1,0 & 4 \\
\hline & Control y operación de equipos & 1,2 & 32 \\
\hline & Media Skills & 1,2 & 0 \\
\hline & Conocimientos de informática & 1,9 & 17 \\
\hline & Procesamiento y análisis de datos & 1,2 & 13 \\
\hline & Comprensión de los procesos y la organización & 1,5 & 20 \\
\hline & $\begin{array}{l}\text { Capacidad de operar con nuevas interfaces } \\
\text { (Integración persona/máquina) }\end{array}$ & 2,2 & 20 \\
\hline & Concientización sobre seguridad informática & 2,1 & 2 \\
\hline
\end{tabular}

Tabla VIII: diferencias entre el valor actual de las competencias técnicas y el valor necesario a 10 años, y la ponderación indicando las competencias a priorizar.

En esta categoría, los resultados muestran que el mayor gap de conocimiento se ubica en la capacidad de operar con nuevas interfaces. Y esta es, a su vez, una de las 3 en las que los empresarios consideran se debería capacitar primero a los trabajadores. Esto implica que en esta área a los empresarios les parece importante que haya una gran

Memoria Investigaciones en Ingeniería, 2019. $\mathrm{N}^{\circ} 17(1) . p p$ 151-171

https://doi.org/10.36561/ING.17.7

ISSN 2301-1092 • ISSN (en línea) 2301-1106 
capacitación en vistas al futuro. A su vez, esta competencia es la que encuentra un valor más alto en un futuro en la escala de 1 a 5 , siendo de 4,2 el puntaje medio que consideran los encuestados que deberán tener sus operarios en un futuro. Es necesario destacar, a su vez, el gap en la concientización sobre seguridad informática, ubicándose casi en primer lugar. Pero se aprecia que los empresarios tienen un muy bajo interés en capacitar en este sentido. Y el tercer mayor gap se encuentra en los conocimientos de informática. Los encuestados comprenden que en un futuro se debe crecer mucho en el nivel de informática que manejan sus trabajadores. Esta percepción muestra una buena comprensión de que en un futuro todos los trabajadores de una forma u otra deberán ser capaces de manejarse con las herramientas informáticas. La competencia que es considerada por la mayoría como la primera en la que enfocar los esfuerzos de recapacitación es en el control y operación de equipos. Pero lo importante a destacar aquí es que los trabajadores ya tienen un buen nivel, por lo que el gap de conocimiento que se deberá sortear es menor. Resulta interesante comentar también los resultados obtenidos para la competencia programación. Como puede verse, los empresarios no le dan mucha importancia a esta competencia, a diferencia de lo que podría suponerse. Esto es porque consideran que los trabajadores medios del sector agropecuario no tienen la necesidad de aprender esta competencia. Indicaron en las entrevistas que en el futuro será necesario que las empresas cuenten con un buen nivel de programación, pero para ello tendrán trabajadores especializados en esta área, formando parte del departamento de IT. Por dicho motivo consideran que no será relevante que los operarios dominen esta competencia.

De este análisis se puede concluir que los principales esfuerzos de recapacitación de los operarios, refiriéndonos a las competencias técnicas, deberían estar enfocados en la capacidad de operar con nuevas interfaces y en conocimientos de informática. Y se deberá tratar de concientizar más a los empresarios acerca de la necesidad de reforzar la seguridad informática, puesto que estas nuevas tecnologías permitirán controlar toda la operativa de la empresa desde internet, por lo que la empresa puede quedar fácilmente expuesta a ataques cibernéticos.

Competencias Metodológicas. En la Figura III se presentan las competencias metodológicas del operario, obtenidas en base a los resultados de la encuesta. Y en la Tabla IX se analizan las diferencias entre el nivel actual y el necesario a 10 años, conjuntamente con la ponderación obtenida por cada competencia.

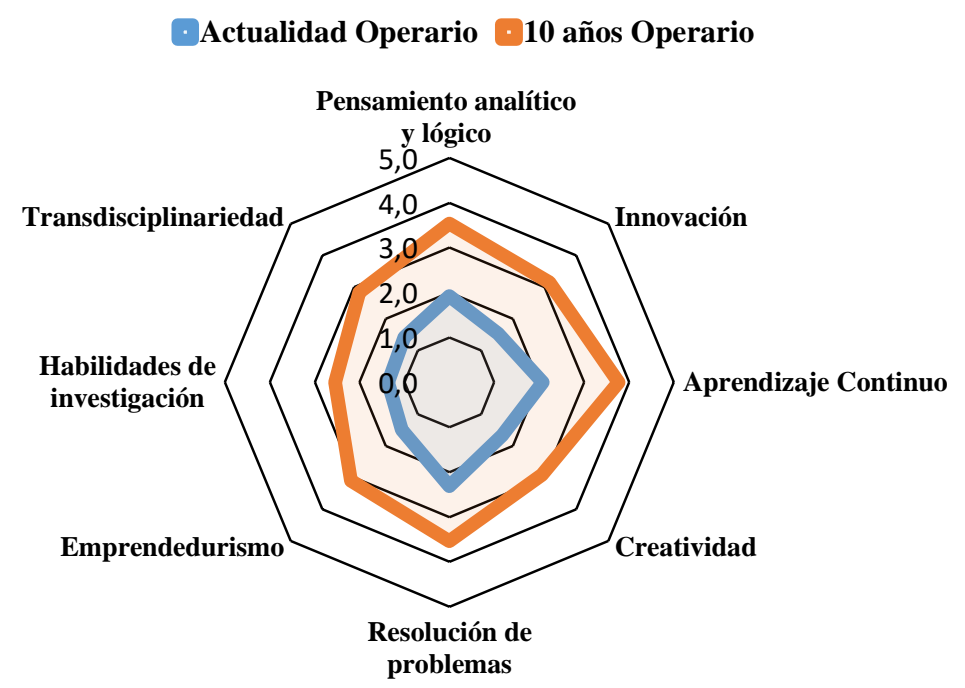

Figura III: Matriz de las competencias metodológicas del operario

Memoria Investigaciones en Ingeniería, 2019. $\mathrm{N}^{\circ} 17(1) . p p$ 151-171

https://doi.org/10.36561/ING.17.7

ISSN 2301-1092 • ISSN (en línea) 2301-1106 


\begin{tabular}{|c|c|c|c|}
\hline & & $\begin{array}{c}\text { Diferencia } \\
\text { Actualidad y } 10 \text { años }\end{array}$ & Ponderación \\
\hline \multirow{8}{*}{ 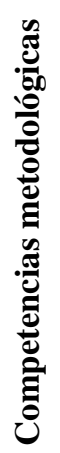 } & Pensamiento analítico y lógico & 1,6 & 24 \\
\hline & Innovación & 1,6 & 9 \\
\hline & Aprendizaje Continuo & 1,7 & 43 \\
\hline & Creatividad & 1,3 & 2 \\
\hline & Resolución de problemas & 1,2 & 16 \\
\hline & Emprendedurismo & 1,6 & 1 \\
\hline & Habilidades de investigación & 1,2 & 5 \\
\hline & Transdisciplinariedad & 1,4 & 7 \\
\hline
\end{tabular}

Tabla IX: diferencias entre el valor actual de las competencias metodológicas y el valor necesario a 10 años, y la ponderación indicando las competencias a priorizar.

De esta categoría, lo que más destaca es la necesidad del aprendizaje continuo. Es el que presenta valor más alto en un futuro, siendo de 3,8 sobre 5. A su vez, es la competencia en la que la diferencia con el nivel actual que poseen los operarios es mayor. Y también es la que presenta la mayor ponderación, muy por encima de la competencia que le sigue dentro del ranking. Esto último indica que los empresarios, en su gran mayoría, consideran que esta competencia será fundamental para el futuro de sus operarios, y están dispuestos a centrar los esfuerzos de capacitación del personal, dentro de esta categoría, en dicha competencia. Estos resultados son coherentes con los de la bibliografía internacional, donde se destaca que, en un futuro, debido a la rapidez con la que las transformaciones se están dando, lo principal que deberán tener los trabajadores será la capacidad de aprender a aprender. En un segundo lugar, los encuestados consideran que se focalizarían en capacitar en pensamiento analítico y lógico. Aprecian que es necesario crecer mucho en esta competencia, siendo la que presenta el segundo mayor gap de conocimiento, estando en este sentido igualada con la innovación. En tercer lugar, consideran que también es importante capacitar en la resolución de problemas. En esta competencia, algo positivo a destacar es que la diferencia con el nivel actual no es excesivamente grande, por lo que la capacitación necesaria no es muy alta.

De estos datos surge que sin duda será prioridad la capacitación en un aprendizaje continuo, en primer lugar. Así lo consideran la mayoría de los encuestados, y ven a su vez que el gap es muy grande. Luego será necesario fomentar el pensamiento analítico y lógico en los empleados. Y también hay que focalizar recursos en desarrollar la resolución de problemas en los operarios. Esta última competencia puede vincularse fácilmente con un desarrollo de la innovación, dado que se puede fomentar a los trabajadores que resuelvan los problemas de una forma creativa que pueda agregarle valor a sus actividades y a la empresa. 
Competencias Sociales. -Dentro de esta categoría, como se aprecia en la Figura IV y en la Tabla X, el nivel actual de todas las competencias es relativamente similar. Las dos que presentan un mayor valor en miras al futuro son trabajo en equipo y capacidad de buena comunicación. Dentro de la matriz, son las dos que llegan a valores más altos dentro de los 10 años. Y, a su vez, son las que presentan un mayor gap frente a la capacidad actual. La literatura internacional también destaca estas competencias como fundamentales en un futuro, por lo que las industrias deberán realizar esfuerzos en potenciarlas dentro de sus empresas $[33,34]$.

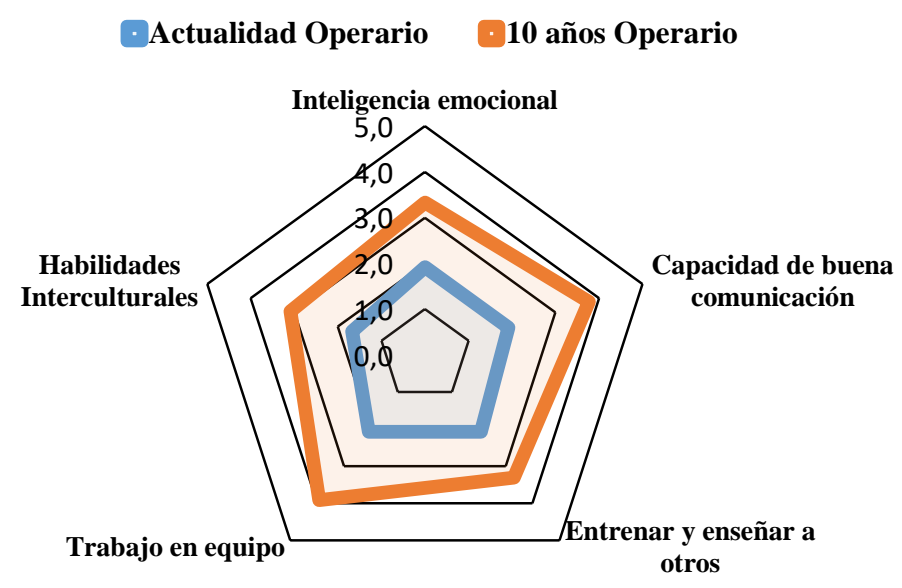

Figura IV: Matriz de las competencias sociales del operario

\begin{tabular}{|c|c|c|c|}
\hline & & $\begin{array}{c}\text { Diferencia } \\
\text { Actualidad y } \\
10 \text { años }\end{array}$ & Ponderación \\
\hline \multirow{5}{*}{ 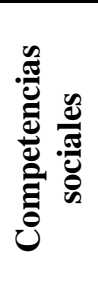 } & Inteligencia emocional & 1,4 & 25 \\
\hline & Capacidad de buena comunicación & 1,8 & 36 \\
\hline & Entrenar y enseñar a otros & 1,2 & 5 \\
\hline & Trabajo en equipo & 1,8 & 31 \\
\hline & Habilidades Interculturales & 1,4 & 1 \\
\hline
\end{tabular}

Tabla X: diferencias entre el valor actual de las competencias sociales y el valor necesario a 10 años, y la ponderación indicando las competencias que los empresarios consideran se enfocarían primero en capacitar.

Frente a la Industria 4.0, en un futuro muchas tareas se automatizarán, y los trabajadores brindarán un verdadero valor agregado en aquellas operativas que sean difícilmente automatizables. Y dentro de las tareas difícilmente automatizables, las competencias que destacan por sobre el resto son las que refieren a aspectos sociales, dado que involucran al trato entre personas, por lo que es muy difícil para una máquina conseguir o imitar dicha competencia. Igualmente se están diseñando robots que buscan emular sentimientos y características propias de las personas, pero dichos avances aún se ubican dentro de un marco experimental. 
Competencias Personales. -Dentro de las competencias personales, lo primero que destaca frente a los otros gráficos, es que hay 2 competencias en las que el nivel actual de los operarios supera al nivel necesario para el futuro, como se observa en la Figura V y en la Tabla XI. Ellas son fuerza física y habilidad manual.

- Actualidad Operario $\cdot 10$ años Operario

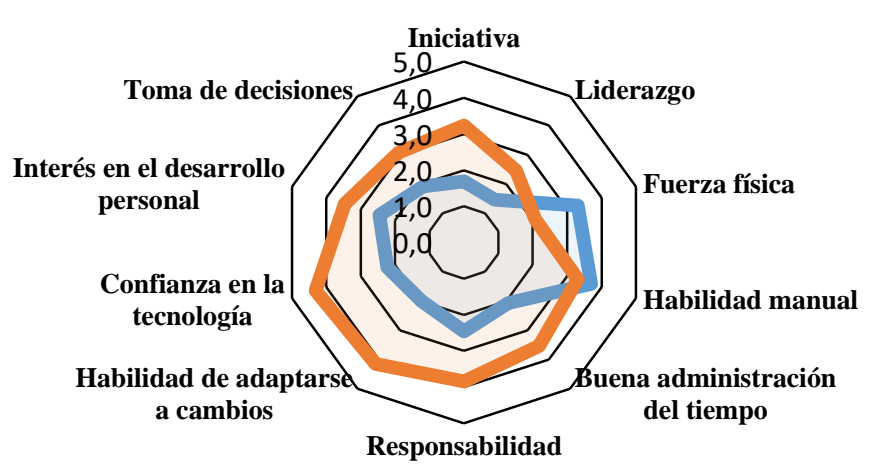

Figura V: Matriz de las competencias personales del operario

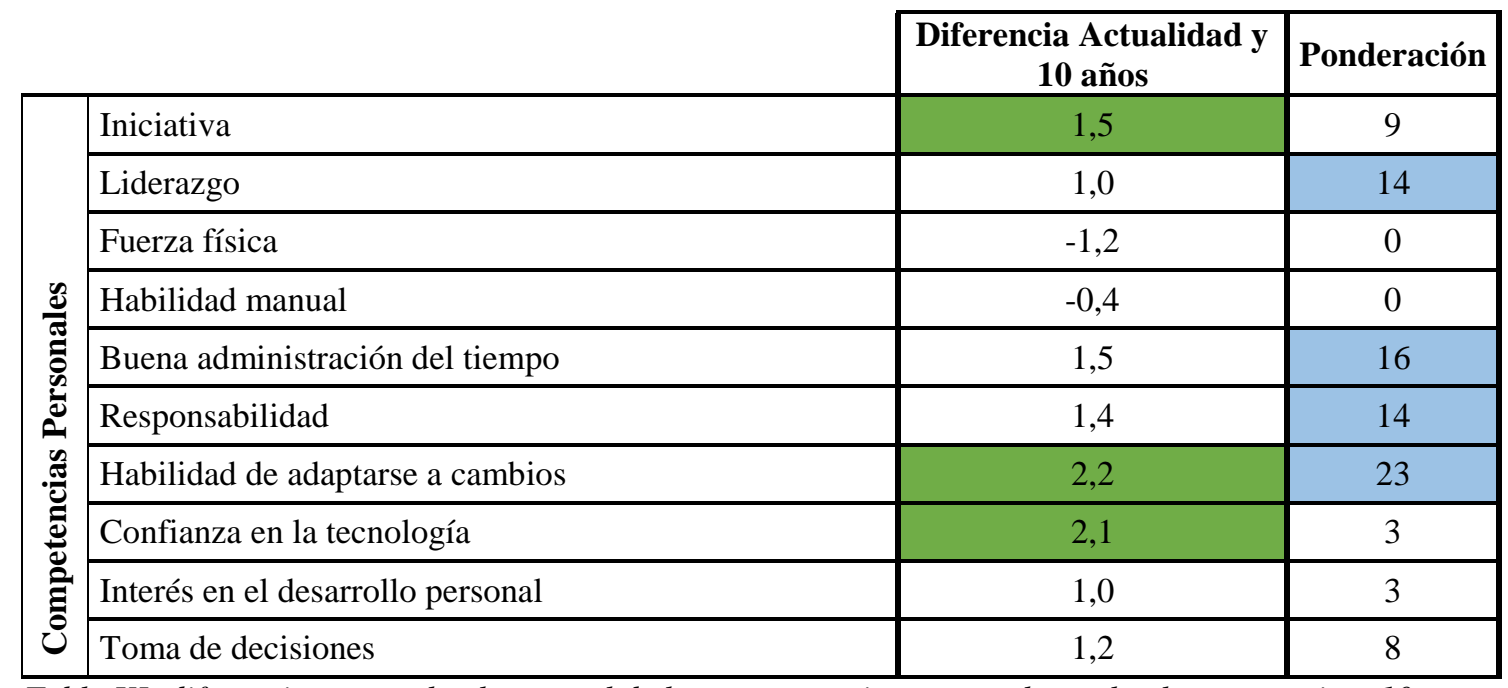

Tabla XI: diferencias entre el valor actual de las competencias personales y el valor necesario a 10 años, y la ponderación indicando las competencias que los empresarios consideran se enfocarían primero en capacitar.

Los resultados obtenidos están alineados con la literatura, donde ninguno de los estudios considera que la fuerza física será una competencia imprescindible frente al futuro, y uno solo hace mención de la necesidad de poseer una buena habilidad manual con el avance de la Industria 4.0. Esto a su vez, es coherente con uno de los objetivos de la aplicación de la Industria 4.0, que es la eliminación de los puestos de trabajo rutinarios y desgastantes físicamente, entre los que se pueden encontrar los trabajos que requieren alta fuerza física.

En referencia a los mayores gaps de competencias, destaca en primer lugar la capacidad para adaptarse a cambios. Esta es, a su vez, la que presenta la mayor

Memoria Investigaciones en Ingeniería, 2019. $\mathrm{N}^{\circ} 17(1) . p p$ 151-171

https://doi.org/10.36561/ING.17.7

ISSN 2301-1092 • ISSN (en línea) 2301-1106 
ponderación. Esta competencia resulta relevante en un futuro, debido a la rapidez con la que se considera que los cambios tecnológicos ocurrirán Por dicho motivo deberán procurar desarrollar sus competencias a una velocidad creciente para poder enfrentarse al cambio [34, 35]. Los trabajadores deberán estar prontos para enfrentarse a cambios en sus profesiones, en el ambiente laboral e incluso en su vida social, y frente a dichas transformaciones, la adaptación al cambio será una herramienta de gran utilidad. La competencia que le sigue en cuanto al gap existente es la confianza en la tecnología, la cual está íntimamente relacionada con la previamente mencionada. Los encuestados advierten que la confianza que los operarios del sector agroindustrial tienen actualmente en las tecnologías es muy bajo, y frente al futuro deberá aumentar al punto que necesitarán contar con una buena capacitación en esta competencia. La necesidad de esta competencia se explica por sí misma, puesto que, si en un futuro gran parte de las tareas estarán automatizadas y los operarios deberán relacionarse diariamente con estas tecnologías, es fundamental que tengan confianza en ella y la utilicen continuamente como parte de su trabajo. A su vez, tanto esta competencia como la anterior, se pueden vincular con la de capacidad de operar con nuevas interfaces, planteada previamente dentro de competencias técnicas. Recordando lo analizado sobre ella, es de las que presenta un mayor gap y un nivel de priorización de los más altos en dicha categoría. Las tres competencias están íntimamente relacionadas, y son de gran importancia en el futuro de la Industria 4.0, la cual estará fundamentalmente caracterizada por la sinergia hombretecnología, tanto dentro de la planta industrial como en la vida diaria de las personas.

6.2.1 Futuro del empleo. -A continuación, se les solicitó a los entrevistados indicar su percepción acerca de cómo iba a variar en los próximos 10 años, la cantidad de trabajadores del sector en 3 niveles distintos: operarios, mandos medios y jefes. Cada encuestado respondió a esta pregunta colocando el foco en su sector de producción. Por lo que, considerando que las entrevistas se realizaron a empresas abarcando diversas áreas del sector, los resultados finales incorporan diversos puntos de vista dentro del sector agropecuario, y por ende hay que tener precaución al intentar generalizar los resultados encontrados. Se les solicitó evaluar, en porcentaje, qué variación de empleo esperaban en esos 3 niveles desde esta fecha a 10 años. Los resultados se presentan en la Tabla XII.

\begin{tabular}{|l|c|}
\hline Puesto de trabajo & Variación en los próximos 10 años (\%) \\
\hline Operarios & $-36 \%$ \\
\hline Mandos medios & $-18 \%$ \\
\hline Jefes & $-13 \%$ \\
\hline
\end{tabular}

Tabla XII: Variación de los puestos de trabajo del sector agroindustrial en los próximos 10 años, basados en los resultados de las entrevistas

Es importante aclarar que, en esta pregunta, las respuestas fueron sumamente variadas, indicando que no hay un consenso generalizado en los empresarios acerca de qué ocurrirá con los puestos de trabajo. Igualmente, es importante destacar que, en promedio, las empresas consideran que en todos los niveles van a tener un descenso de los trabajadores. El descenso más marcado estará en los operarios, con una estimación de más de un tercio de los mismos perdiendo su empleo en los próximos 10 años. Estos números son a tener en consideración, dada la cantidad de trabajadores que esta industria representa.

Analizando las respuestas de forma más individual, en referencia a los operarios, todos los entrevistados indicaron que consideran que en el futuro va a descender la cantidad de trabajadores de esta categoría en el sector. El valor más extremo indicado fue de un descenso de 75\%, y el menor valor fue de un descenso del 10\%. Mencionaron que,

Memoria Investigaciones en Ingeniería, 2019. $\mathrm{N}^{\circ} 17(1)$. pp 151-171

https://doi.org/10.36561/ING.17.7

ISSN 2301-1092 • ISSN (en línea) 2301-1106 
actualmente, los altos costos de la automatización no permiten que estos valores sean superiores.

En opinión de uno de los entrevistados, las empresas líderes en países desarrollados tienen una visión más amplia y creativa de cómo van a impactar las nuevas tecnologías y cómo aprovechar al máximo sus beneficios, por lo que esas industrias pueden expandir su modelo de negocios, y consecuentemente, necesitar de más empleados. Destacó, entre otros ejemplos, los cultivos hidropónicos automáticos y la carne de laboratorio como opciones para que una empresa diversifique su producción y amplíe así su necesidad de trabajadores. Indicó que, si las empresas agroindustriales del país se enfocaran en cómo poner la tecnología en aras de nuevas invenciones y nuevas líneas de negocio, entre las que se pueden contar las mencionadas anteriormente, o una sofisticación de los productos, entonces los resultados podrán incluso llevar a un aumento de la mano de obra.

Por último, varios entrevistados destacaron que los puestos que más se verán afectados en sus empresas no serán tanto relacionados con el agro en sí, sino relacionados más a los servicios y administración de la empresa. Indicaron de ejemplo las pérdidas en áreas administrativas tales como los pagos de sueldo y liquidaciones de impuestos. Expresaron que procuran que el personal que ya no sea necesario en un área pueda ser reconvertido dentro de otra área de la misma empresa donde pueda brindar un mayor valor, pero plantearon que esto no siempre puede ser realizado. A su vez, destacaron la importancia de las nuevas competencias: "lo que más se va a necesitar dentro de la empresa son las competencias blandas" y otro entrevistado acotó "el contacto cara a cara con el productor va a seguir estando, la confianza es vital". Finalmente, en relación a los puestos que más refieren a la industria del agro en sí, indicaron que las transformaciones de mano de obra están dadas por las nuevas tecnologías tales como cosechadoras automáticas, salas de ordeñe robotizadas, ganadería de precisión, maquinaria en la industria forestal, entre varios ejemplos que comentaron. Y que esta transformación afectará principalmente a la mano de obra menos calificada, y por consiguiente requerirán personal con mejores competencias en el uso de herramientas tecnológicas.

6.2.2 Colaboración Universidad/Empresa. Por último, se procuró conocer la opinión de los entrevistados acerca de la necesidad de establecer una mayor colaboración entre la universidad y la empresa. Las miradas obtenidas fueron principalmente dos. Por un lado, el $88 \%$ de los encuestados considera que las universidades deberían colaborar con las empresas para formar estudiantes más preparados para las nuevas realidades del mundo laboral, y a su vez colaborar en la recalificación de los trabajadores existentes. Por otro lado, el $12 \%$ de los entrevistados planteó que la tarea de recualificación de los trabajadores correspondía principalmente a las empresas y otros agentes educativos y gubernamentales (y no tanto a la universidad en sí), y que la misión principal de la universidad debería ser la de generar y formar mentes y profesionales preparados para enfrentar lo más diversos y nuevos desafíos.

Algunos entrevistados mencionaron que realizar una colaboración entre ambas partes era algo que resultaría muy importante para el tiempo actual. Recomendaron realizar en conjunto proyectos de desarrollo y adopción de tecnología, utilizando los conocimientos de la universidad para crear herramientas que fueran realmente útiles para la realidad actual de las empresas. Por ejemplo, indicaron que sería muy útil desarrollar la robótica industrial a nivel universitario, y que las facultades de ingeniería deberían estar realizando más cursos relacionados con esta área. Algunos remarcaron que la universidad está en deuda en fomentar un emprendedurismo tecnológico de automatización que sería de gran utilidad para las empresas. En el mismo sentido, destacaron la necesidad de tener 
más graduados que posean herramientas de programación, por lo que recomiendan que las universidades actualicen las materias que brindan dentro de sus cursos y procuren brindarles un foco más tecnológico.

Otros encuestados recomiendan que las universidades se esfuercen en mayor medida en brindarles a sus estudiantes herramientas útiles para el mundo del trabajo, y que se centren en fomentar las competencias profesionales tales como aprendizaje continuo. Aconsejan, también, que la universidad aporte en concientizar a la clase política acerca de la urgencia de prepararse frente a los cambios que se avecinan.

En este sentido, todos los entrevistados concuerdan en la necesidad de generar espacios de colaboración entre las empresas, el gobierno y las universidades, para así poder aunar esfuerzos en pro del desarrollo nacional futuro. Indicaron que esto es algo que ya ocurre en los países más desarrollados, pero que en Uruguay hay aún una gran falencia al respecto. En este sentido, las empresas detectan una falencia, donde la universidad trabaja mucho en la creación de conocimiento teórico, pero no logran adoptar esos conocimientos a la realidad de las industrias. Si se implementan estos espacios de sinergia permitirá que las universidades detecten con mayor rapidez qué competencias requieren las industrias, de forma de poder ajustar su plan de estudios para satisfacer dicha demanda en un tiempo adecuado.

En particular, varios entrevistados indican que, si bien ya existen algunas iniciativas aisladas en este sentido, no advierten que exista un plan de dirección firme para unificar todas las iniciativas y que puedan trabajar de forma conjunta. "Falta lograr la integración de los diversos actores y organismos que colaboran en este sentido".

6.2.3 Comentarios finales de los entrevistados. -Uno de los elementos finales que destacaron los empresarios en las entrevistas son los problemas educativos a los que se enfrenta el país. En este sentido, varios señalaron que la educación debería transformarse, centrándose más en metodologías que fomenten el "aprender haciendo" (learning by doing) y también el "aprender a aprender". Indican que durante su vida laboral en las empresas han advertido que los empleados adquieren mejor las competencias que necesitan si las aprenden mediante la experiencia. "Lo vivencial es clave, y más si considerás la necesidad de trabajar en equipo" decía uno de los entrevistados. Sugieren que estas técnicas deberían ser más fomentadas dentro de la educación, tal vez siendo necesaria una adaptación de los planes de estudio y un enfoque más basado en la enseñanza de competencias y no tanto en la enseñanza de contenidos. En relación a esto, algunos entrevistados observaron que recapacitar y re enseñar a los empleados actuales de más edad puede presentar retos todavía mayores. Parte de sus empleados dentro de la empresa no tienen las capacidades de leer ni escribir, por lo que proponen que el aprendizaje se base más en actividades didácticas tales como metodologías prácticas de aprendizaje basado en juegos formativos y trabajo entre pares. Recomiendan estudiar qué métodos de enseñanza son más acordes para trabajadores de más edad con niveles educativos bajos. Dentro de esta misma línea, señalan que, aunque se implementen medidas para capacitar a sus operarios, una dificultad que se presenta es evaluar el retorno de dichas acciones. Indican que es muy difícil estimar la relación costo/beneficio de las actividades de capacitación de las personas. Por lo que también proponen que sería interesante poseer más herramientas en este sentido.

Por último, señalan que todos los trabajadores de la empresa, desde los gerentes hasta los operarios deben comprender realmente que estos desafíos tecnológicos ya están presentes, y que deberán trabajar en conjunto para evitar las pérdidas de puesto de trabajo y los riesgos asociados.

Memoria Investigaciones en Ingeniería, 2019. $\mathrm{N}^{\circ} 17(1) . p p$ 151-171

https://doi.org/10.36561/ING.17.7

ISSN 2301-1092・ISSN (en línea) 2301-1106 
6. Discusión y Conclusiones. -Este proyecto tiene como objetivo analizar las competencias necesarias en el futuro de la Industria 4.0 en el sector agroindustrial, y el nivel que las empresas consideran que será necesario alcanzar para un futuro. Luego de una extensa revisión bibliográfica, se construyó una matriz con 31 competencias agrupadas en cuatro categorías, la cuales fueron luego validadas y priorizadas en base a entrevistas realizadas a 15 stakeholders del sector agroindustrial uruguayo.

A partir de los resultados de las entrevistas, se generaron gráficos de radar identificando el gap de competencias necesario para avanzar en materia de transformación digital y tecnológica en el sector. Las competencias determinadas como las más relevantes para el futuro son: Conocimientos de Informática, Capacidad de operar con nuevas interfaces, Aprendizaje Continuo, Pensamiento Analítico y Lógico, Expresión Oral, Trabajo en equipo, Confianza en la tecnología y Flexibilidad.

Los resultados obtenidos de las entrevistas fueron consistentes con la bibliografía internacional, mostrando que las competencias consideradas más importantes para la transformación de la Industria 4.0 en la agroindustria concuerdan con la mayoría de las mismas competencias necesarias para las otras industrias encontradas en la bibliografía.

En este sentido, el proyecto espera contribuir a llenar el gap de conocimiento acerca de las competencias necesarias para el sector agroindustrial, y de esta manera, aportar información para ayudar en la recapacitación de los trabajadores para así reducir el impacto de la Industria 4.0 en los puestos de trabajo. Futuras líneas de investigación deberían ser enfocadas en el análisis de las competencias principales en otras industrias y de esta manera generar más evidencias para poder indicar si existe un conjunto de competencias generales que todo trabajador del futuro debe tener.

En relación a los cambios que se avecinan en el sector agroindustrial, la Industria 4.0 está aquí y es necesario prepararse. Frente a estos cambios se presentan dos caminos. Por un lado, retrasar el avance hasta que no sea posible seguir resistiendo, o anticiparse y prever los cambios posibles de forma de adelantarse y obtener el mayor provecho.

Relacionado con este segundo enfoque, se pueden presentar algunas recomendaciones adicionales para una buena adaptación de la Industria 4.0. En particular, los stakeholders entrevistados indicaron que el problema de recapacitación es difícil de resolver y por ende se deben tomar distintas medidas.

En primer lugar, varios estudios mencionan que la preparación para estas nuevas tecnologías debe ser realizada de forma conjunta por las empresas, el gobierno y los educadores. No deben ser tres entidades trabajando de forma independiente, sino que debe haber una gran colaboración entre las partes para que los resultados sean los mejores ([1, $20,26,33,36,37]$. Uno de los principales objetivos de esta colaboración debe ser el desarrollo de un aprendizaje continuo en las personas, para que sean capaces de adaptarse con más facilidad frente a los cambios. Esto debe ser realizado mediante programas conjuntos de desarrollo de competencias, tanto dentro de las empresas como a través de las instituciones educativas formales [19].

De forma complementaria, frente al futuro, será necesario considerar también el desarrollo de un proceso continuo de adquisición de nuevas competencias y conocimientos. Se recomienda la aplicación de programas cortos de desarrollo de competencias específicas, principalmente en áreas de tecnología y ciencias, así como en creatividad, pensamiento crítico y resolución de problemas [35, 36]. Gran parte de este éxito estará dado en la capacidad del gobierno, empresas y educadores en anticipar qué competencias serán imprescindibles para el futuro, y centrar los esfuerzos de recapacitación en ellas. Que las empresas sean capaces de desarrollar una organización

Memoria Investigaciones en Ingeniería, 2019. №17(1). pp 151-171

https://doi.org/10.36561/ING.17.7

ISSN 2301-1092・ISSN (en línea) 2301-1106 
con una cultura que impulse a los trabajadores a aprender es visto como una herramienta importante para tener éxito [34].

Por otro lado, para mantener la competitividad, las empresas deben acelerar sus esfuerzos en el camino de la Industria 4.0. Es necesario que los líderes empresariales consideren la mejor manera de reubicar la mano de obra que automaticen, ya sea dentro de sus organizaciones o fuera de ellas, tanto para su propio desempeño como para ser una empresa socialmente responsable [35]. Por lo tanto, los programas de reentrenamiento y de mejora de las competencias de los trabajadores dentro de las empresas serán fundamentales. En particular, estudios internacionales recomiendan recapacitar a los propios empleados, los cuales ya conocen el funcionamiento de la empresa y pueden aportar mayor valor [29]. De esta forma se está brindando un desarrollo profesional y humano a muchos trabajadores, con lo que se está mejorando tanto la propia empresa, como la sociedad en su conjunto. En este sentido, las empresas, más que considerar la automatización como una forma de ahorrar en costos laborales, deben verla como una oportunidad de creación de valor, buscando que se potencie la sinergia hombre-máquina, alcanzando así un verdadero progreso.

Por su parte, el gobierno tendrá la misión de facilitar el relacionamiento entre los diversos stakeholders, para generar espacios de sinergia y oportunidades de innovación. A su vez, deberá procurar generar una coordinación de las iniciativas que promuevan la creación de trabajo, para que todos los agentes interesados puedan trabajar juntos de forma que los programas individuales se apoyen mutuamente y no se superpongan. En paralelo, el gobierno deberá estimular el crecimiento de la economía y la incorporación de nuevas herramientas tecnológicas por parte de las empresas, de forma de posibilitar una mayor generación de empleo. Deberá fomentar, además, que las empresas tengan programas de entrenamiento propios, y debe colaborar en la generación de relaciones estratégicas entre las empresas y las instituciones educativas, de forma que los cambios en educación sean lo más ágiles y efectivos posibles para adecuarse a un mundo del trabajo donde los cambios ocurrirán cada vez con mayor velocidad. También debe conseguir generar un mercado de trabajo más fluido, procurando que los trabajadores puedan conseguir puestos de trabajo con más rapidez.

Por último, las instituciones educativas deben estar dispuestos a adaptar sus planes de estudio de forma ágil, para que no se forme una brecha entre las competencias que se desarrollan en el ámbito educativo y las requeridas a nivel profesional En este mismo aspecto, el informe del World Economic Forum [10] indica que el 65\% de los alumnos ingresando actualmente al nivel de educación primario, terminarán trabajando en empleos completamente nuevos que actualmente no existen, y que aproximadamente el 50\% del conocimiento que un estudiante adquiere en el primer año de facultad estará desactualizado para el momento que el alumno se gradúe. En este sentido, algunos estudios proponen que las universidades deberán centrarse en brindar programas universitarios más enfocados en capacidades de procesamiento de datos y herramientas informáticas. Las competencias blandas también deberán tener su foco durante el desarrollo universitario, de forma de promover la innovación, la colaboración entre pares, el trabajo en equipo y el relacionamiento social de los futuros profesionales [29, 31]. Un modelo educacional que se propone como beneficioso para el futuro es el modelo dual alemán, donde la enseñanza teórica y práctica se combina para obtener mejores resultados. A su vez, parte de este aprendizaje se da fuera de los lugares distintos a los tradicionales, con lo que se fomenta el concepto del aprendizaje continuo dentro de las empresas y en la vida diaria [29].

Memoria Investigaciones en Ingeniería, 2019. $\mathrm{N}^{\circ} 17(1) . p p$ 151-171

https://doi.org/10.36561/ING.17.7

ISSN 2301-1092・ISSN (en línea) 2301-1106 
En resumen, si los países de todo el mundo fallan en la percepción de los riesgos que presenta la llegada de la Industria 4.0, especialmente en el sector agropecuario, y no fortalecen la capacitación de sus trabajadores con un modelo que les permita enfocar eficazmente los esfuerzos, este sector se verá fuertemente afectado por la pérdida de empleos, con muchos trabajadores quedando fuera del mercado laboral en los próximos años. Es indudable que grandes beneficios derivarán de la Industria 4.0, pero los riesgos implicados no deben ser subestimados. Por eso, y considerando la importancia de esta industria a nivel global, y los altos riesgos de automatización de sus puestos de trabajo, más estudios relacionados con la necesidad de recapacitación de los trabajadores, y la manera de realizarla, se vuelven esenciales para el futuro bienestar de la sociedad en su conjunto.

\section{Referencias}

[1]. Benešová, A. y Tupa, J. (2017) «Requirements for Education and Qualification of People in Industry 4.0», Procedia Manufacturing, 11(June), pp. 2195-2202

[2]. Manyika, J., Lund, S., et al. (2017) «Jobs Lost, Jobs Gained : Workforce Transitions In A Time Of Automation», McKinsey Global Institute.

[3]. Deloitte LLP (2015) «From brawn to brains. The impact of technology on jobs in the $\mathrm{UK»,p.16.}$

[4]. Slusarczyk, B. (2018) «Industry 4.0 - Are We Ready?», Polish Journal of Management Studies, 17.

[5]. Frey, C. B., y Osborne, M. A. (2017) «The future of employment: How susceptible are jobs to computerisation?», Technological forecasting and social change, 114, pp. 254280.

[6]. Hamzeh, R., Zhong, R. y Xu, X. W. (2018) «A Survey Study on Industry 4.0 for New Zealand Manufacturing», Procedia Manufacturing. Elsevier B.V., 26, pp. 49-57.

[7]. Lorenz, M. et al. (2016) «Time to Accelerate in the Race Toward Industry 4.0», The Boston Consulting Group. Recuperado a partir de https://www. bcgperspectives. com/Images/BCG-Time-to-Accelerate-in-the-Race-Toward-Industry-4.0-May2016_tcm80-211060.pdf

[8]. Rojko, A. (2017) «Industry 4 . 0 Concept: Background and Overview», International Journal of Interactive Mobile Technologies (iJIM), 11(5), pp. 77-91.

[9]. Hermann, M., Pentek, T. y Otto, B. (2016) «Design principles for industrie 4.0 scenarios», Proceedings of the Annual Hawaii International Conference on System Sciences. IEEE, 2016-March, pp. 3928-3937.

[10]. World Economic Forum (2016) «The Future of Jobs Employment, Skills and Workforce Strategy for the Fourth Industrial Revolution», Global Challenge Insight Report, World Economic Forum.

[11]. Aulbur, W., CJ, A. y Bigghe, R. (2016) «Skill Development for Industry 4.0», Roland Berger, BRICS Skill Development Working Group, India Section, pp. 1-50.

[12]. World Bank (2018) «Agriculture and Food» Disponible en: http://www.worldbank.org/en/topic/agriculture/overview (Acceso: 20/02/2019).

[13]. Uruguay XXI. Informe agropecuario. 2018;0-46.

[14]. Uruguay XXI. Estadísticas de comercio exterior. 2018.

[15]. Ackermann, M. N. y Cortelezzi, Á. (2017) «Mercado de trabajo agropecuario : situación y prospectiva hacia el Uruguay Agrointeligente de 2030», Anuario OPYPA 2017.

[16]. Munyo, I. (2016) «Y por casa cómo andamos?», Revista de negocios del IEEM, 19(3), pp. 22-27.

[17]. Hecklau, F. et al. (2016) «Holistic Approach for Human Resource Management in Industry 4.0», Procedia CIRP. The Author(s), 54, pp. 1-6.

[18]. Lleó, A. et al. (2017) «Competencias Profesionales 4.0».

Memoria Investigaciones en Ingeniería, 2019. №17(1). pp 151-171

https://doi.org/10.36561/ING.17.7

ISSN 2301-1092 • ISSN (en línea) 2301-1106 
[19]. World Economic Forum (2018) «The Future of Jobs Report».

[20]. Gehrke, L. et al. (2015) «A Discussion of Qualifications and Skills in the Factory of the Future: A German and American Perspective», VDI/ASME Industry, 4, pp. 1-28.

[21]. Smit, J. et al. (2016) «Industry 4.0», Parlamento Europeo.

[22]. Erol, S. et al. (2016) «Tangible Industry 4.0: A Scenario-Based Approach to Learning for the Future of Production», Procedia CIRP. Elsevier B.V., 54, pp. 13-18.

[23]. Breuer, Z. (2012) «Agriculture, forestry and fishing: sector skills assessment 2012», London: UKCES.

[24]. European Centre for the Development of Vocational Training (2017) «People, Machines , Robots And Skills», (July), pp. 1-4.

[25]. Moldovan, L. (2018) «State-of-the-Art Analysis on the Knowledge and Skills Gaps on the Topic of Industry 4 . 0 and the Requirements for Work-Based Learning in Romania», 15[1], pp. 32-35.

[26]. Störmer, E. et al. (2014) «The Future of Work : Jobs and skills in 2030».

[27]. Scott, C. L. (2015) «El futuro del aprendizaje 2 ¿Qué tipo de aprendizaje se necesita en el siglo XXI?».

[28]. Kergroach, S. (2017) «Industry 4.0: New Challenges and Opportunities for the Labour Market», 11(4), pp. 6-8

[29. Lorenz, M. et al. (2015) «Man and Machine in Industry 4.0 How Will Technology Transform the Industrial Workforce Through 2025?», The Boston Consulting Group.

[30]. Cotet, G. B., Balgiu, B. A. y Zaleschi, V. C. (2017) «Assessment procedure for the soft skills requested by Industry 4.0», MATEC Web of Conferences, 121, p. 07005.

[31]. Davies, A., Fidler, D. y Gorbis, M. (2011) «Future Work Skills 2020», Institute for the Future for University of Phoenix Research Institute, 2011, vol. 540.

[32]. Gómez, J. L. P. (2002). «Estrategias de ponderación de la respuesta en encuestas de satisfacción de usuarios de servicios» Metodología de encuestas, 4(2), 175-193.

[33]. Motyl, B. et al. (2017) «How will Change the Future Engineers' Skills in the Industry 4.0 Framework? A Questionnaire Survey», Procedia Manufacturing, 11 pp. 1501-1509.

[34]. Deloitte (2018) «The Fourth Industrial Revolution is here - are you ready?», Report. UK: Deloitte Insight, p. 20

[35]. Manyika, J., Chui, M., et al. (2017) «A Future That Works : Automation, Employment, And Productivity», McKinsey Global Institute Research, Tech. Rep

[36]. Rüßmann, M. et al. (2015) «Industry 4.0: The Future of Productivity and Growth in Manufacturing Industries», Boston Consulting Group, 9, pp. 54-89.

[37]. Baena, F. et al. (2017) «Learning Factory: The Path to Industry 4.0», Procedia Manufacturing, 9, pp. 73-80. 